\title{
Evaluation of the substitutions in 212, 342 and 215 amino acid positions in binding site of organophosphorus acid anhydrolase using the molecular docking and laboratory analysis
}

\author{
Heidari $\mathrm{MF}^{1}$, Arab SS${ }^{2}$, Noroozi-aghideh $\mathrm{A}^{3}$, Tebyanian $\mathrm{H}^{1,4}$, Latifi $\mathrm{AM}^{1}$ \\ Applied Biotechnology Research Center, Baqiyatallah University of Medical Sciences, Tehran, Iran. \\ amlatify@yahoo.com
}

\begin{abstract}
OBJECTIVE: Organophosphorus Acid Anhydrolase (OPAA) is used as one of the most important enzymes in the decontamination process of organophosphate compounds. In this study, we aimed to evaluate the effects of amino acid substitution in OPAA's substrate-binding site on its catalytic activity using the rational engineering strategy. METHODS: The native and three mutant forms of OPAA enzyme including 4ZWP, 4ZWU and Mut6 were studied using the docking technique toward parathion, paraoxon and R-VX compounds. Furthermore, enzyme assay was performed on the native OPAA and Mut6 toward parathion.

RESULTS: Docking results showed a decreased catalytic activity of the mutant forms toward parathion and paraoxon. Furthermore, enzyme assay showed in accordance with docking results a decreased activity of Mut6 compared to the native form. The results of docking prediction for R-VX showed an increased catalytic activity of $4 Z W P$ and $4 Z W U$. $4 Z W U$ had the highest activity, while the activity of Mut6 was lower than the native form. CONCLUSION: Amino acid positions of 212 and 342 seem to be important sites in the small pocket of OPAA affecting the enzyme catalytic activity. Therefore, substitution of these sites with appropriate amino acids depending on the substrate structure, can affect the enzyme catalytic efficiency (Tab. 2, Fig. 3, Ref. 30). Text in PDF www.elis.sk. KEY WORDS: organophosphorus acid anhydrolase (OPAA), enzyme activity, molecular docking.
\end{abstract}

\section{Introduction}

Industrial and scientific development has improved human health, which increased the usage of biocides and pesticides (1). Organophosphate compounds are highly toxic substances that act as pesticides by irreversible inhibition of acetylcholinesterase enzyme in the nervous system (2). High toxicity of these factors led to their use as chemical weapons (3). Growing use of organophosphate compounds resulted in environmental pollutions and therefore, many researches were performed on decontamination methods $(4,5)$. Different chemical and physical methods were used to decontaminate the organophosphate compounds, which caused adverse effects on the environment $(4,6)$. Bioremediation has recently emerged as a new method for removing these compounds $(7,8)$.

Detection of enzymes in various organisms, such as: bacteria, squid and mammals, which were able to neutralize organophos-

${ }^{1}$ Applied Biotechnology Research Center, Baqiyatallah University of Medical Sciences, Tehran, Iran, ${ }^{2}$ Department of Biophysics, Faculty of Biological Science, Tarbiat Modares University, Tehran, Iran, ${ }^{3}$ Department of Hematology, Faculty of Paramedicine, Aja University of Medical Sciences, Tehran, Iran, and ${ }^{4}$ Research Center for Prevention of Oral and Dental Diseases, Baqiyatallah University of Medical Sciences, Tehran, Iran

Address for correspondence: A.M. Latifi, Applied Biotechnology Research Center, Baqiyatallah University of Medical Sciences, Tehran, Iran. Phone: +98.21.88617712, Fax: +98.21.82482549 phate compounds opened a new path of biodegradation methods (9). Many types of these enzymes belong to the Phosphotriesterases family (10). The most important enzymes in this group are Organophosphorus Acid Anhydrolase (OPAA), Organophosphorus Hydrolase (OPH) and Diisopropil Flourophosphatase (DFPase) the one isolated from the Alteromonas, Flavobacterium and Loligo Vulgaris species, respectively (11).

OPAA is a bacterial prolinase enzyme with phosphotriesterase activity (12). This enzyme was initially identified in 1946 by Abraham Mazur in rabbit tissue (13). Later in 1991, OPAA enzyme was detected and purified from the Halophilic Alteromonas strain JD6.5 by DeFrank and Cheng (14). By identifying this enzyme, attempts were made to isolate it from other bacterial strains $(15,16)$. Considering the importance of this enzyme in the development of enzyme-based decontamination processes, many researches were performed to evaluate its properties and mechanism of action, and its optimum performance conditions were determined $(13,17)$. Crystallography of this enzyme was performed for the first time by Vyas et al, in 2010 (13).

In 2013, Stepankova et al, determined the structure of OPAA enzymes from Alteromonas macleodii by X-ray Crystallography. She showed that OPAA enzymes isolated from the strain JD6.5 were different from the ones from strain Macleodii, having a molecular weight of $58 \mathrm{kDa}$ and two amino and carboxy domains (14).

The OPAA is one of the most well-known enzymes with pita bread folding and two manganese ions in the active site (13). 


\section{9-143}

The functional form of this enzyme is oligomer, which is tetramer a dimer of dimers. The active site of the enzyme is composed of H336, H381, D244, E420 and D255 residues. Like other phosphotriesterases, this enzyme has three pockets in the substrate binding site as well, including the small, large and leaving group pockets. Residues Y212, V342, H343 form the small pocket that is capped by $\mathrm{D} 45^{\prime}$ from the opposite subunit in the dimer. The large pocket is formed by residues L225, H226, H332, R418 and capped by W89' from the other subunit. The third pocket is very small and consists of two residues F292 and L366. By forming a hydrogen bond with a free phosphoryl oxygen in substrate, H343 helps to position the substrate for catalysis (18). The activity of OPAA from the strain JD6.5 was determined in a number of organophosphate warfare agents and it was shown that Soman (GD) had the highest activity of $3145 \mathrm{kcat}(\mathrm{s}-1)$ and R-VX agent had the lowest activity of 1.8 kcat (s-1) $(19,20)$.

As a new therapeutic strategy, the use of these enzymes was considered for the treatment of poisoning with organophosphate compounds (21). If the lower amounts of enzyme could be able to neutralize the higher amounts of organophosphate compounds, it indicates a higher catalytic efficiency of the enzymes (22). Each of OPAA enzymes exhibits different levels of activity for organophosphate compounds. Several research efforts have been made to improve the activity of these enzymes (18).

Several approaches have been developed to improve the enzyme activity, based on the change of the enzyme environment such as: $\mathrm{pH}$, temperature and ionic strength of the buffer, and change of the amino acid sequence (23). Making modifications in gene sequence led to the advent of genetic/enzyme engineering strategies. Protein engineering can involve either one of two general strategies including the random and rational approaches (24). In rational approach, detailed information on three-dimensional structure and the relationship between structure and function is required for selecting target sites for engineering (25). Effect of the mutations on the enzyme function can be evaluated by the in silico studies (23).

Daczkowski et al for the first time in 2015 showed an increase of OPAA catalytic efficiency toward R-VX by substituting three amino acids at the OPAA's substrate-binding site. They introduced some of the mutated forms of enzyme OPAA such as: 4ZWP and 4ZWU with an improved activity toward R-VX. (19).

In this study, we aimed to evaluate the effect of amino acid substitution in OPAA's substrate-binding site on its catalytic activity using the rational engineering strategy.

Using the molecular docking method, three substrates belonging to the two groups of organophosphates including the parathion and paraoxon from P-O group, and the R-VX from P-S group were evaluated in the presence of 4 enzymes including one native enzyme, 4ZWP and 4ZWU mutated enzymes, and an engineered enzyme. Then, using bioinformatics studies, the effects of mutation on the structure and function of the enzyme were investigated. Finally, based on these results, the engineered enzyme was investigated using site direct mutation technique and its activity was experimentally tested in the presence of parathion as an available substrate.

\section{Materials and methods}

\section{Bioinformatics studies}

DNA sequence of the OPAA enzyme (GenBank: U29240.1) was obtained from https: //www.ncbi.nlm.nih.gov/genbank. The protein sequences were taken from the Protein Database at http: //www.uniprot.org (UniProtKB - Q44238 (PEPQ_ALTSX)). The secondary and tertiary structures of the protein and PDB 3L7G files for the wild type, and 4ZWP and 4ZWP mutated forms were extracted from http: //www.ebi.ac.uk/pdbe. To generate a new mutated pdb file, the protein sequence was loaded into I-TASSER and mutated pdb file was obtained. The three-dimensional structure of the protein was analyzed using Swiss Pdb-Viewer, Molegro molecular viewer and YASARA software. PubChem was used to prepare the PDB file for substrates. AutoDock Tools V 1.5.6 was used for molecular docking between the ligand and the enzyme.

\section{Docking}

According to instruction of the AutoDock Tools, the grid parameter file (gpf) value for all docking processes was set to 126 for $\mathrm{x}, \mathrm{y}$ and $\mathrm{z}$ dimensions, and the spacing values (angstrom) were also set to less than 0.2 (between 0.18-0.19). Center Grid box was also prepared using the above setting and selecting the amino acids involved in the active site. The grid boxes were set based on the position of amino acids, particularly in the small, large and leaving pockets to cover all of these positions.

The docking parameter file (dpf) was also selected by choosing the genetic algorithm parameter number was set to $150 \mathrm{GA}$ run, and other default settings were not changed. The gpf, dpf and pdbqt files were uploaded to the server of Bioinformatics Department of the Faculty of Life Sciences at Tarbiat Modares University at http: //bioinf.modares.ac.ir/. Docking results were sorted into different clusters and the ones with the best ligand binding in the active site of the receptor were selected. Then, ligand-receptor complexes were analyzed by YASARA software $(26,27)$.

\section{Laboratory analysis}

Latifi et al, expressed OPH and DFPase enzymes using an amino acid anchoring domain system of Ice Nucleation Protein (INP) in suitable strains of E. coli (28-30). In another study by this group, the InaV-N-OPA recombinant protein was cloned using pCDFDuet-1 vector in E. coli BL21 DE3 PlysS and INP anchoring system was inserted at the beginning of OPAA sequence (unpublished results).

\section{Designing the mutations}

To create point mutations, the QuikChange II XL Site-Directed Mutagenesis Kit, Agilent Technologies, was chosen and, in accordance with its instructions, was taken to the design of the primer. Mutagenic primers were designed using QuikChange Primer De-

Tab. 1. The sequence of the designed primers used for mutagenesis.

\begin{tabular}{ll}
\hline Name & Sequence (5' to 3') \\
\hline a635t_a643t_t644a_ & $5^{\prime}$-attaagcgcgacatagttgccgaaagcgttgtcattctcgctgtg-3' \\
\hline
\end{tabular}




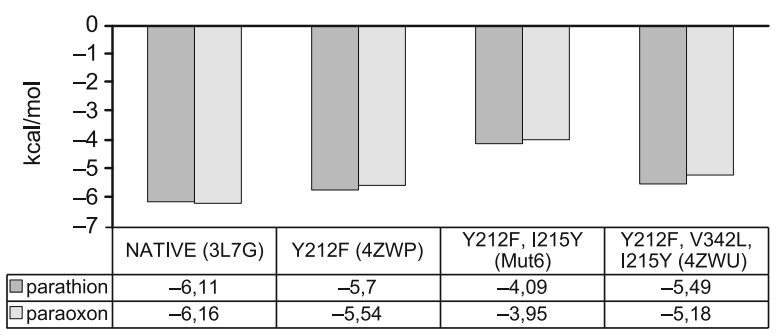

Fig. 1. Docking results for the native and mutated forms of OPPA enzyme toward parathion and paraoxon.

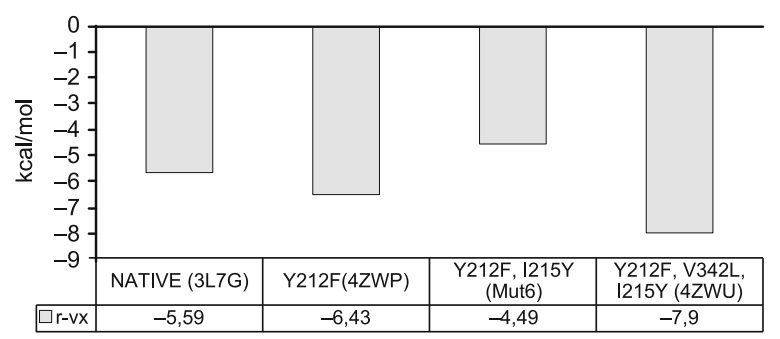

Fig. 2. Docking results for the native and mutated forms of OPPA enzyme toward R-VX compound.

sign Program (www.agilent.com/genomics/qcpd). The sequences of the designed primers are shown in Table 1.

\section{Performing the mutation}

At first, bacteria harboring OPAA gene were cultured in sterile conditions. Then, plasmid extraction was performed using the GeneAll ${ }^{\circledR}$ ExprepTM Plasmid Kit and plasmid concentration was determined using a Nanodrop ND-1000 Spectrophotometer (NanoDrop Technologies). According to the kitss instruction, PCR was performed to create the mutant form and then, PCR product was treated with Dpn I for 1 hour at $37^{\circ} \mathrm{C}$. Finally, a recombinant vector (Mut 6) harboring a mutated form of OPPA was achieved, in which tyrosine and isoleucine at positions 212 and 215 were substituted by phenylalanine and tyrosine, respectively.

\section{Transformation of the vector containing the mutation}

The transformation process was carried out using the heat shock method on competent E.coli cells. These bacteria were transferred to a plate containing streptomycin and incubated for 24 hours in $37^{\circ} \mathrm{C}$ and then, evaluated for colony growth by transfected bacteria as streptomycin-resistant cells.

One of the colonies was transferred to LB broth with $0.05 \mathrm{mM}$ IPTG to induce recombinant protein expression. Then, protein expression was assessed by SDS-PAGE technique.

\section{Enzyme assay}

Activity of the native and mutated enzymes, which was expressed at the host cell surface, was evaluated toward Parathion (Sigma-Aldrich). The tests were performed in triplicate. Cells harboring the mutant vector were harvested and used to assay enzyme activity based on spectrophotometry. At first, cells were washed with Tris-HCL $75 \mathrm{mM}$ buffer and diluted to set as unit cell density (OD $600=1)$. For each assay, $800 \mu \mathrm{l}$ of Tris-HCL buffer, $100 \mu \mathrm{l}$ of $\mathrm{MnCl} 2(0.1 \mathrm{M})$ and $100 \mu \mathrm{l}$ of cells suspension were mixed. Then, $1 \mu \mathrm{l}$ of parathion $(1 \mathrm{~g} / \mu \mathrm{l})$ was added. Absorbance was measured at $\mathrm{OD}_{410}$ after 3 minutes incubation and one unit of OPAA activity was defined as millimoles PNP produced per minute (29).

\section{Results}

In this study using the molecular docking method, two forms of OPPA enzyme including the native and mutated forms were evaluated toward the three substrates and the corresponding binding free energies were assayed. The best docking for each substrate was selected according to the correct pattern of placement in the binding site and position of the enzyme's active site.

Docking prediction results for the native and mutated forms of OPPA enzyme toward the two substrates belonging to the P-O group including parathion and paraoxon are shown in Figure 1.

Docking prediction results for the native and mutated forms of OPPA enzyme towards a substrate belonging to the P-S group (R-VX) are shown in Figure 2.

Enzyme assay results for the native and a mutant form (Mut6) of OPPA enzyme towards parathion are shown in Table 2.
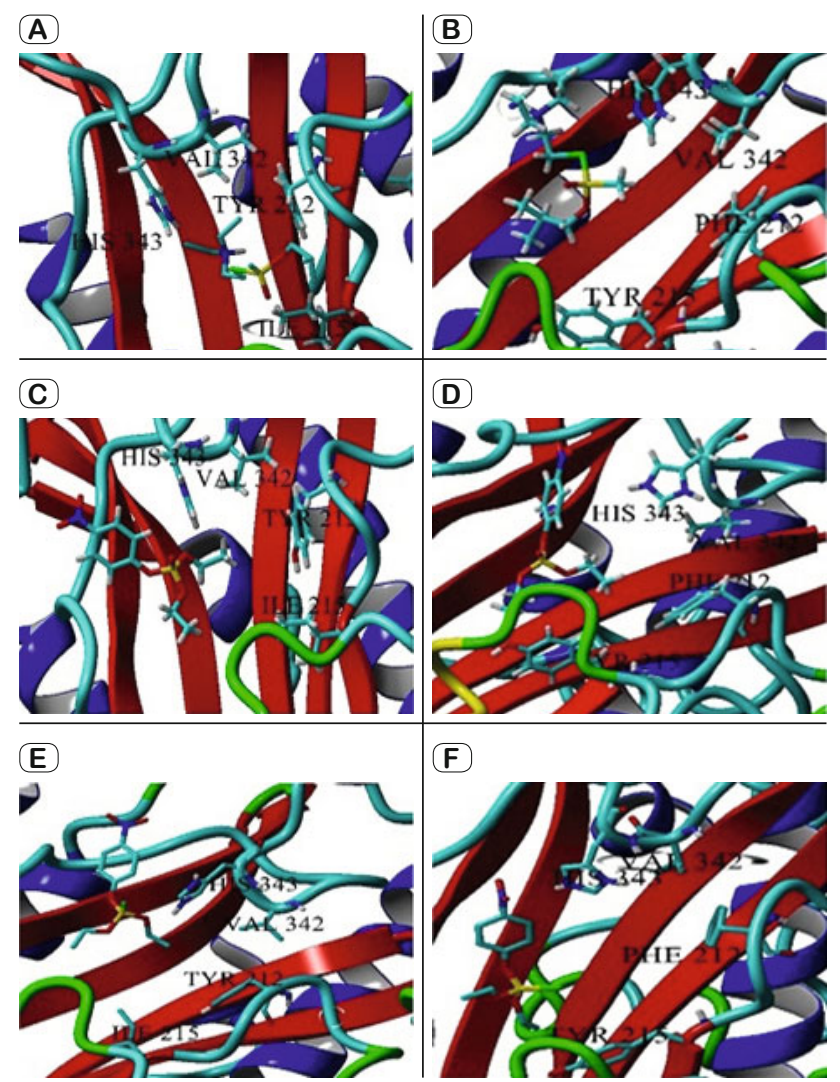

Fig. 3. Docking results for the binding sites native OPAA and Mut6. A: Native OPAA toward R-VX; B: Mut6 toward R-VX; C: Native OPAA toward paraoxon; D: Mut6 toward paraoxon; E: Native OPAA toward parathion; F: Mut6 toward parathion. 
Tab. 2. Enzyme assay for OPAA toward parathion.

\begin{tabular}{ll}
\hline Enzyme & Enzyme activity \\
\hline Native & $26.66 \pm 1.5 \mathrm{U} / \mathrm{mL}$ \\
Mut6 & $6.46 \pm 1.3 \mathrm{U} / \mathrm{mL}$ \\
B13 & $0.0 \pm 0.3$ \\
\hline
\end{tabular}

Native: a wild-type OPPA enzyme; Mut6: a mutant form of OPPA enzyme; B13: vector-free host cell

Images corresponding to the docking results for the native and Mut6 toward the three substrates are shown in Figure 3.

\section{Discussion}

The only previous report on mutations of OPAA enzyme was performed by Daczkowski et al, that led to a 30 -fold increase in catalytic activity toward R-VX. Based on their results, creating the mutation in the small pocket could have a higher effect on the enzyme activity than in large and leaving group pockets. Therefore, in this study, we evaluated the effects of amino acid substitutions in the small pocket of OPAA enzyme on its catalytic activity using the docking method and enzyme assay.

According to the critical role of H343 in forming the hydrogen bond with the free phosphoryl oxygen in the small pocket that helps to position the substrate for catalysis, we didn>t consider this amino acid site for substitution. For this reason, we evaluated the effects of substitution of two other amino acid positions in the small pocket (Y 212 and V 342) and I 215 between the small and large pockets.

Here we confirmed the previous results of enzyme assay on 4ZWP and 4ZWU mutant forms of OPAA using the docking method. On the other hand, we created a mutant form (Mut6) of OPAA and evaluated its activity using enzyme assay toward parathion and docking study. Both approaches in our study consistently showed a decreased activity of this form.

In 4ZWP, the substitution of a polar amino acid (Tyr) with a non-polar one (Phe) at position $212(\mathrm{Y} 212 \mathrm{~F})$ led to increase in the hydrophobicity of the enzyme binding site. In Mut6, the substitution of a non-polar amino acid (Ile) with a polar one (Tyr) at position 215 (I215Y), in addition to Y212F led to a change in the hydrophobic pattern of the enzyme binding site. In 4ZWU, the substitution of Val with Leu as two non-polar amino acids at position 342 (V342L) in addition to Y212F and I215Y was performed. Therefore, the difference between the catalytic activity of 4ZWU and Mut6 might be related to the larger size of Leu than Val and special position of the 342 beside H343.

The results of docking prediction for 4ZWP, 4ZWU and Mut6 toward parathion and paraoxon showed that the mentioned substitutions in enzyme binding site led to a decrease in its catalytic activity. 4ZWP and 4ZWU had equal catalytic activity, while Mut6 had the least activity. Furthermore, enzyme assay showed in accordance with docking prediction a decreased activity of Mut6 compared to the native form.

Accordingly, it can be concluded, that the optimal activity of the enzyme towards the P-O substrates, was achieved in circumstances with the minimal changes compared to the native form.
The results of docking prediction for R-VX showed an increased catalytic activity of 4ZWP and 4ZWU. 4ZWU had the highest activity, while the activity of Mut6 was lower than the one in the native form.

In full agreement with our results of docking prediction, Daczkowski et al, demonstrated that the catalytic activity (k cat (s-1) of 4ZWP and 4ZWU toward R-VX increased from 1.8 to 11 and 32 , respectively.

In conclusion, our results indicated that amino acid positions of 212 and 342 are important sites in the small pocket of OPAA that affect the enzyme catalytic activity. Therefore, substitution of these sites with appropriate amino acids depending on the substrate type and structure, can affect the enzyme activity.

\section{References}

1. Zwanenburg B, Mikolajczyk M, Kielbasinski P. Enzymes in Action Green Solutions for Chemical Problems: Springer Science \& Business Media; 2012.

2. Singh BK. Organophosphorus-degrading bacteria: ecology and industrial applications. Nat Rev Microbiol 2009; 7 (2): 156-164.

3. Delfino RT, Ribeiro TS, Figueroa-Villar JD. Organophosphorus compounds as chemical warfare agents: a review. J Braz Chem Soc 2009; 20 (3): 407-428.

4. Ortiz-Hernández ML, Sánchez-Salinas E, Olvera-Velona A, FolchMallol JL. Pesticides in the environment: impacts and their biodegradation as a strategy for residues treatment. Pesticides-formulations, effects, fate InTech 2011: 978-953.

5. Tebyanian H, Mirhosseiny SH, Kheirkhah B, Hassanshahian M. Isolation and Identification of Mycoplasma synoviae From Suspected Ostriches by Polymerase Chain Reaction, in Kerman Province, Iran. Jundishapur JMicrobiol 2014; 7 (9): e19262.

6. Shakeri F, Latifi AM, Mirzaii M, Babavalian H, Tebyanian H. Introduction of fungal necrosis inducing phytotoxin for biocontrol of Sinapis arvensis as a common weed in Iran. J Anim Plant Sci 2017; 27 (5): $1702-1710$

7. Porto ALM, Melgar GZ, Kasemodel MC, Nitschke M. Biodegradation of pesticides. Pesticides in the modern world - pesticides use and management InTech 2011: 407-438.

8. Tebyanian H, Hassanshahian M, Kariminik A. Hexadecane-degradation by Teskumurella and Stenotrophomonas strains isolated from hydrocarbon contaminated soils. Jundishapur J Microbiol 2013; 6 (7): e9182.

9. SARNAIK SS. Biodegradation of organophosphorus pesticides. Proc Indian natn Sci Acad 2004; 1: 57-70.

10. Zheng Y, Long L, Fan Y, Gan J, Fang J, Jin W. A review on the detoxification of organophosphorus compounds by microorganisms. Afr J Microbiol Res 2013; 7 (20): 2127-2134.

11. Farnoosh G, Latifi AM. A review on engineering of organophosphorus hydrolase (OPH) enzyme. JABR 2014; 1 (1): 1-10.

12. Mazur A. An enzyme in animal tissues capable of hydrolyzing the phosphorus-fluorine bond of alkyl fluorophosphates. J Biol Chem 1946; 164 (1): 271-289.

13. Vyas NK, Nickitenko A, Rastogi VK, Shah SS, Quiocho FA. Structural insights into the dual activities of the nerve agent degrading organophosphate anhydrolase/prolidase. J Biochem 2010; 49 (3): 547-559. 
14. DeFrank JJ, Cheng T-C. Purification and properties of an organophosphorus acid anhydrase from a halophilic bacterial isolate. J Bacteriol 1991; 173 (6): 1938-1943.

15. DeFrank JJ, Beaudry WT, Cheng T-C, Harvey SP, Stroup AN, Szafraniec LL. Screening of halophilic bacteria and Alteromonas species for organophosphorus hydrolyzing enzyme activity. Chem-Biol Interact 1993; 87 (1-3): 141-148.

16. Cheng T-C, Harvey SP, Stroup AN. Purification and properties of a highly active organophosphorus acid anhydrolase from Alteromonas undina. Appl Environ Microbiol 1993; 59 (9): 3138-3140.

17. Taherian A, Fazilati M, Moghadam AT, H T. Optimization of purification procedure for horse $\mathrm{F}\left(\mathrm{ab}^{\prime}\right) 2$ antivenom against Androctonus crassicauda (Scorpion) venom. Trop J Pharm Res 2018; 17 (3): 409-414.

18. Bigley AN, Raushel FM. Catalytic mechanisms for phosphotriesterases. Biochim Biophys Acta 2013; 1834 (1): 443-453.

19. Daczkowski CM, Pegan SD, Harvey SP. Engineering the Organophosphorus Acid Anhydrolase Enzyme for Increased Catalytic Efficiency and Broadened Stereospecificity on Russian VX. J Biochem 2015; 54 (41): 6423-6433.

20. Cheng T-c, DeFrank JJ, Rastogi VK. Alteromonas prolidase for organophosphorus G-agent decontamination. Chem-Biol Interact 1999; 119: 455-462.

21. Masson P. Catalytic bioscavengers: the new generation of bioscavenger-based medical countermeasures. Handbook of Toxicology of Chemical Warfare Agents (Second Edition): Elsevier; 2015. p. 1107-1123.
22. Allahyari H, Latifi AM. Diisopropyl-fluorophosphatase as a Catalytic Bioscavenger. JABR 2017; (4): 477-482.

23. Hibbert EG, Dalby PA. Directed evolution strategies for improved enzymatic performance. Microb Cell Fact 2005; 4 (1): 29.

24. Turanli-Yildiz B, Alkim C, Cakar ZP. Protein engineering methods and applications. Protein Eng: InTech; 2012.

25. Damborsky J, Brezovsky J. Computational tools for designing and engineering enzymes. Curr Opin Chem Biol 2014; 19: 8-16.

26. Kim H-H, Hyun J-S, Choi J, Choi K-E, Jee J-G, Park SJ. Structural ensemble-based docking simulation and biophysical studies discovered new inhibitors of Hsp90 N-terminal domain. Sci Rep 2018; 8 (1): 368.

27. El-Desoky E-SI, Keshk EM, El-Sawi AA, Abozeid MA, Abouzeid LA, Abdel-Rahman A-RH. Synthesis, biological evaluation and in silico molecular docking of novel 1-hydroxy-naphthyl substituted heterocycles. Saudi Pharm J 2018.

28. Karami A, Latifi AM, Khodi S. Comparison of the organophosphorus hydrolase surface display using InaVN and Lpp-OmpA systems in Escherichia coli. J Microbiol Biotechnol 2014; 24 (3): 379-385.

29. Latifi AM, Karami A, Khodi S. Efficient surface display of Diisopropylfluorophosphatase (DFPase) in E. coli for Biodegradation of Toxic Organophosphorus Compounds (DFP and Cp). Appl Biochem Biotechnol 2015; 177 (3): 624-636.

30. Latifi AM, Khajeh K, Farnoosh G, Hassanpour K, Khodi S. The cytoplasmic and periplasmic expression levels and folding of organophosphorus hydrolase enzyme in Escherichia coli. Jundishapur J Microbiol 2015; 8 (12). 